\title{
Removal of Metallic and Organic Contaminants from Aqueous Streams by Novel Filtration Methods
}

Progress Report to the US. Department of Energy

Grant Number: DE-FG07-96ER14688

Nelly M. Rodriguez

Chemistry Department, Northeastern University, Boston, MA 02125

Tel (617) 3732085

Fax (617) 3738795

\section{INTRODUCTION}

Graphite nanofibers, are a novel material that has been developed in our laboratory from the metal catalyzed decomposition of certain hydrocarbons (1). These structures possess a cross-sectional area that varies between 5 to $100 \mathrm{~nm}$ and have lengths ranging from 5 to $100 \mathrm{~mm}$ (2). High-resolution transmission electron microscopy studies have revealed that the nanofibers consist of extremely well-ordered graphite platelets (3), which are oriented in various directions with respect to the fiber axis. The arrangement of the graphene layers can be tailored to a desired geometry by choice of the correct catalyst system and reaction conditions, and it is therefore possible to generate structures where the layers are stacked in a "ribbon", "herring-bone", or "stacked" orientation.

An example of the later structure is shown in the high resolution electron micrograph, Figure la, where the lines across correspond to individual planes of graphite that are separated at a minimum distance of $0.34 \mathrm{~nm}$. The structural features of the nanofiber can be better appreciated in the schematic renditions, Figures $1 \mathrm{~b}$ and $1 \mathrm{c}$. The unique combination of small cross-sectional area, which is estimated to be on average 20 $\mathrm{nm}$, and the abundance of exposed edges makes the material an ideal solid for adsorption. The suitability of the material for the selective adsorption of a variety of molecules is illustrated in Figure 1c, where it can be seen that adsorption could be achieved upon access of the molecule to the inner regions of the solid. One the most outstanding features of graphite nanofibers, is that the solid consists entirely of non-rigid wall nanopores that extend across the entire solid.

The process for the synthesis of graphite nanofibers produced from the decomposition of hydrocarbons and carbon monoxide over selected metal surfaces at temperatures over the range 450 to $700^{\circ} \mathrm{C}$ has been optimized and it is possible to produce relatively large quantities of high purity material in short periods of time ca. 100 grams per hour. These structures have been found to exhibit unique behavior when exposed to hydrogen at elevated pressures and moderate temperatures.

\section{EXPERIMENTAL METHODS}

Graphite nanofibers of various conformations were prepared according to procedures that have been described previously $(1,2)$ and characterized using transmission electron microscopy. Nitrogen surface area was determined at $77 \mathrm{~K}$ in a 100CX Omnisorp ${ }^{\mathrm{TM}}$ apparatus. 
In the first part of this project the suitability of graphite nanofibers for the adsorption of selected organic molecules from aqueous solutions was tested by allowing a solution of organic to interact with selected samples of GNF. In a typical experiment, $0.2 \mathrm{~g}$ of the carbonaceous solid was deposited in a solution containing $0.5 \mathrm{~mL}$ of a ethyl or butyl alcohol in $60 \mathrm{~mL}$ of deionized water. The material was continuously stirred and maintained at room temperature for up to 160 hours. Care was taken to prevent loss of the alcohol due to evaporation by keeping the container covered. The uptake of alcohol by the carbon was monitored as a function of time by taking samples of the solution at various periods of time that were analyzed by Gas Chromatography. Identical experiments were conducted using an active carbon sample possessing a surface area $\sim 7.5$ times that measured for GNF.

\section{RESULTS AND DISCUSSION}

Transmission electron micrograph examination of the product indicated that the material consisted entirely of fibers possessing a highly ordered conformation. These studies indicated that fibers were the only product of the reaction, i.e. no nanoparticles or other forms of carbon were detected. BET surface area measurements indicated that the surface area of the GNF was $120 \mathrm{~m}^{2} \cdot \mathrm{g}^{-1}$, whereas the active carbon sample exhibited a value of $750 \mathrm{~m}^{2} \cdot \mathrm{g}^{-1}$.

In the first series of adsorption experiments changes in the concentration of ethanol were monitored as a function of time in the presence of GNF and active carbon at room temperature are presented in Figure 2. It can be seen that the ability of GNF to adsorb the alcohol far exceeds that of active carbon despite the larger value of surface area found for the latter material. After a period of about 60 hours the uptake of ethanol on active carbon was $6 \%$, whereas under the same conditions a value of $38 \%$ was achieved with nanofibers. It could be concluded that although active carbon has a much larger surface area, the adsorption behavior of the organic from an aqueous solution is non-selective; in other words pores in active carbon get quickly saturated with water leaving little room for the alcohol. On the other hand, due to the chemical nature of GNF consisting entirely of graphite platelets, their interaction with the organic is much stronger than with water which results in a selective adsorption. It is important to realize that in the "as-received" form the edges of the nanofibers are hydrophobic in nature and as a consequence, are expected to exhibit adsorption characteristics that are favorable towards the uptake of organic molecules.

In a second series of reactions the removal of butanol from an aqueous solution was investigated on both types of carbon materials and these data are presented in Figure 3. From these plots it is evident that once again the graphite nanofibers exhibit a superior performance for adsorption of the alcohol than that displayed by the active carbon. In this case maximum adsorption capacity of butanol on carbon nanofibers is established after a period of 75 hours and under these conditions complete removal of the alcohol from solution is achieved. In contrast, when active carbon was used as the adsorbate only about $22 \%$ of the butanol is taken up. Furthermore from a comparison of the data in Figures 2 and 3 it would appear that with either of the carbon materials, the adsorption of butanol from an aqueous solution is a more facile process than that of 
ethanol from the corresponding mixture. This conclusion was substantiated in experiments where the solution contained equal amounts of both alcohols and once again butanol was preferentially adsorbed on the nanofibers and active carbon.

In a further set of experiments we are probing the influence of various pretreatments of the nanofibers on the adsorption characteristics. Initial studies have focused on the differences realized from immersion of the material for 60 hours in nitric and hydrochloric acids, respectively. Inspection of the data shown in Figure 4 reveals that these two treatments induce dramatic differences in the subsequent adsorption characteristics of the nanofibers towards the two alcohols. While immersion in $10 \%$ hydrochloric acid is an essential step to activate the nanofibers towards adsorption of alcohols from water, the corresponding treatment in nitric acid effectively destroys this property. It is possible that reaction in nitric acid results in an interaction that culminates in the edge regions of the graphite platelets in the nanofibers becoming hydrophilic in nature and in the presence of excess water the selective adsorption of alcohol is prevented.

\section{TENTATIVE CONCLUSIONS}

Carbon nanofibers possessing a high degree of crystalline order have been found to exhibit excellent adsorption characteristics for the removal of small amounts of alcohol from aqueous solution. A comparison with active carbon shows that even though the nanofibers have a surface area that is 7 times lower their performance for this separation process is far superior to that displayed by the former material. A further feature to emerge from this work is the finding that pre-treatment conditions has a crucial effect on the subsequent adsorption behavior; while prolonged immersion in hydrochloric was found to be extremely beneficial, a similar treatment in nitric acid virtually nullified the advantages of using nanofibers for the process.

\section{FUTURE STUDIES}

In the coming year we plan to exploit the potential of highly graphitic nanofibers as a selective adsorption agent for the removal of trace amounts of metals from aqueous solutions. Taking advantage of the high electrical conductivity coupled with a high value of surface area to volume in graphite nanofibers we intend to use the material as an electrode for the electrochemical removal of metallic contaminants from water. The efficiency of GNF possessing surface areas ranging from 50 to 600 will be tested.

\section{REFERENCES}

1. M. S. Kim, N. M. Rodriguez and R. T. K. Baker, J. Catal. 131, 60 (1991).

2. $\quad$ N. M. Rodriguez, J. Mater. Res. 8, 3233 (1993).

3. N. M. Rodriguez, A. Chambers and R. T. K. Baker, Langmuir 11, 3862 (1995). 


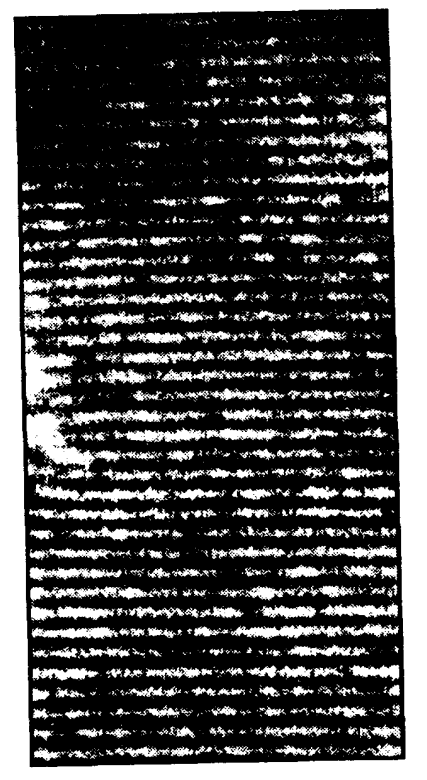

a

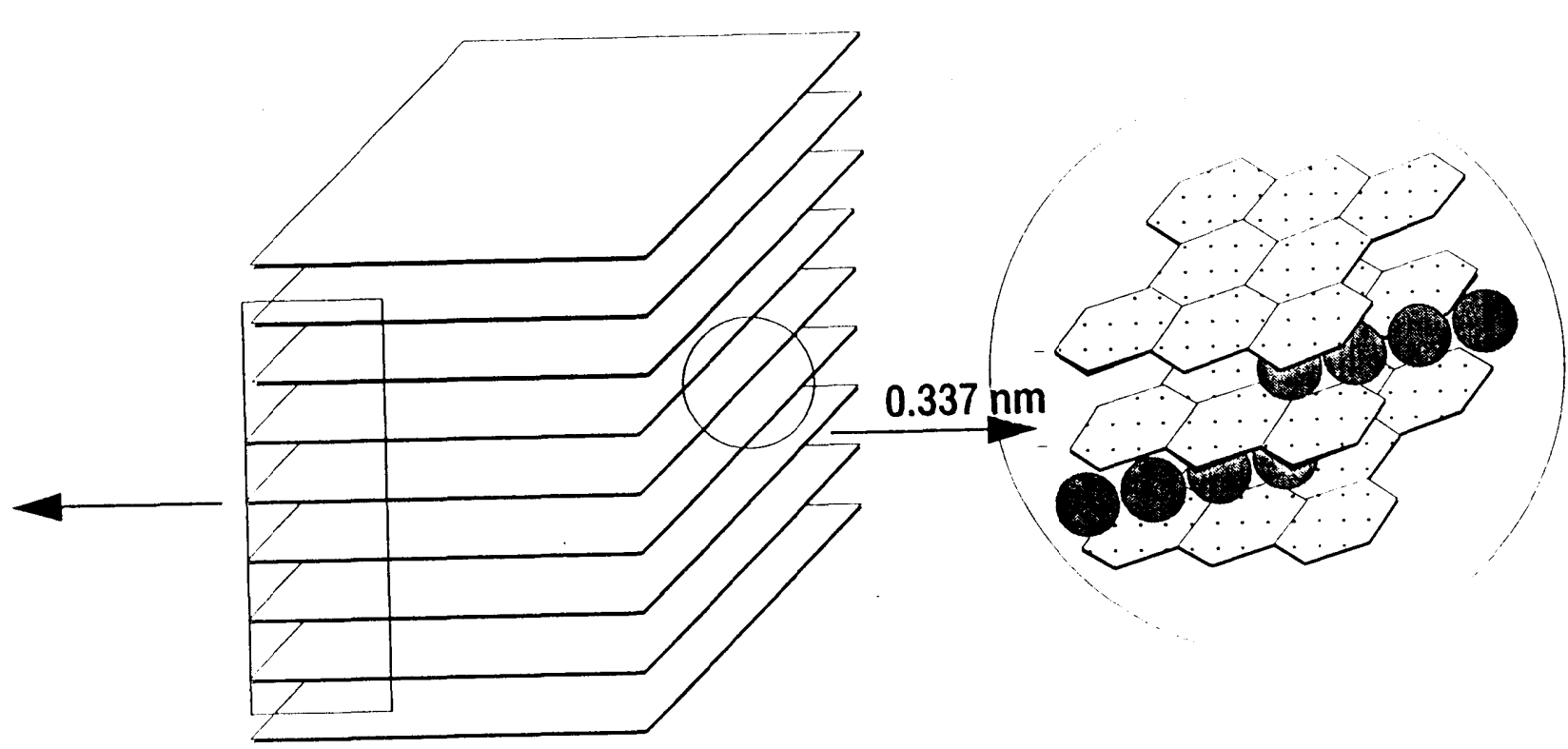

b
C

Figure 1. (a) Transmission electron micrograph of "stacked" Graphite Nanofiber (GNF); (b) schematic representation of nanotiber illustrated in (a); and detail of proposed adsorption of organic molecules in GNF. 
Figure 2. Adsorption of Ethanol on Carbons

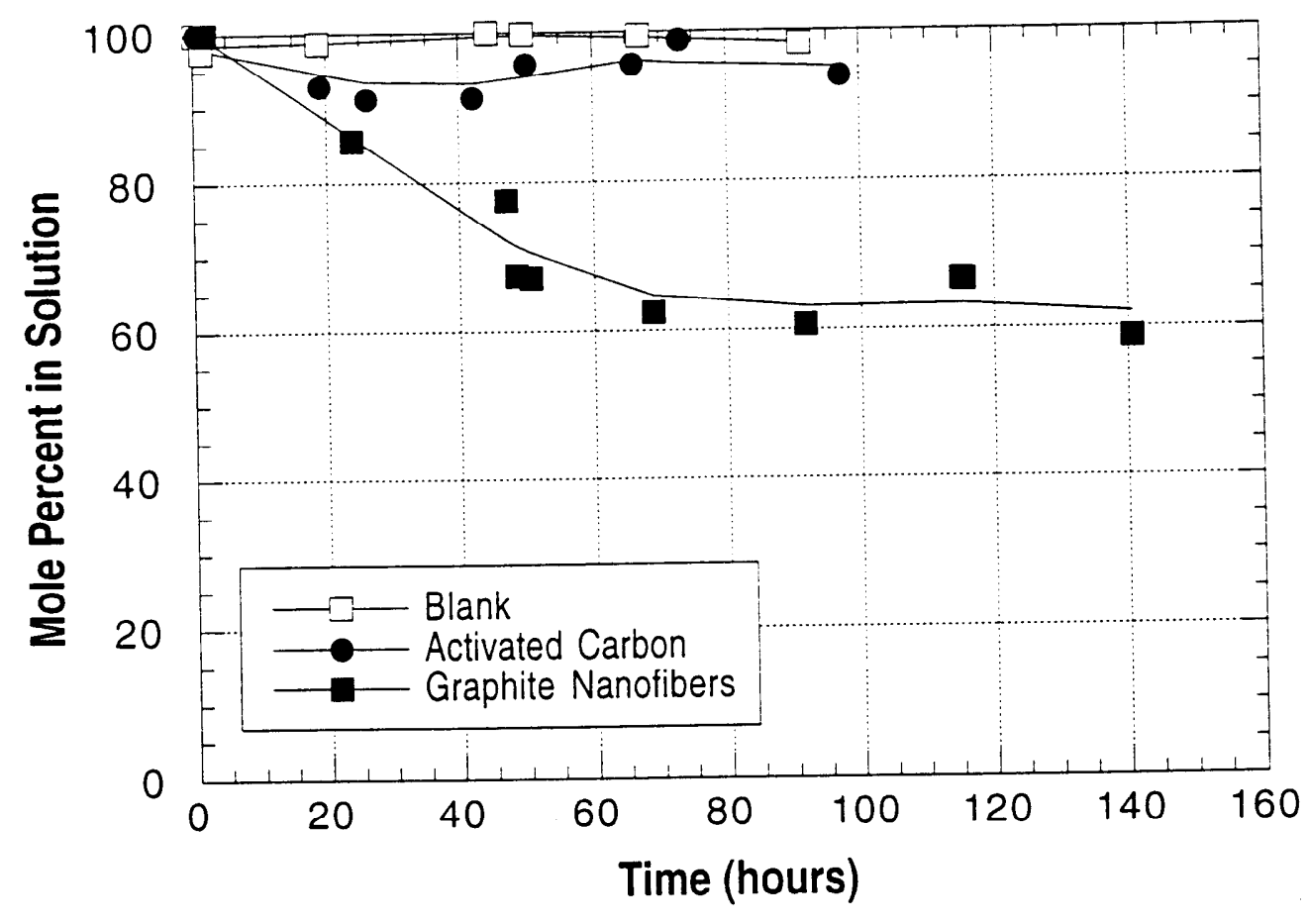

Figure 3. Adsorption of Butanol on Carbons

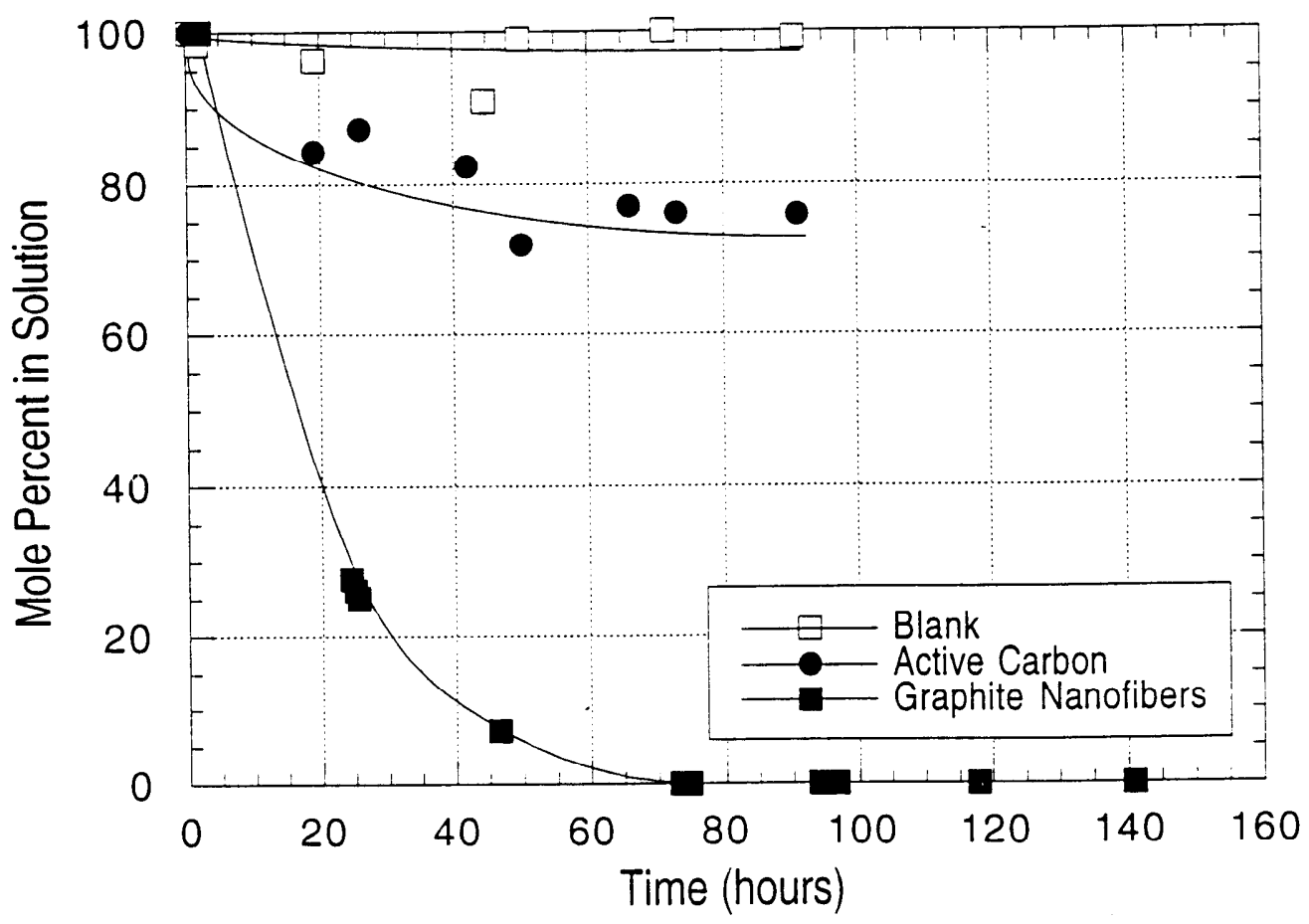


Figure 4. Effect of Acid Treatment on Adsorption of Alcohols on GNF

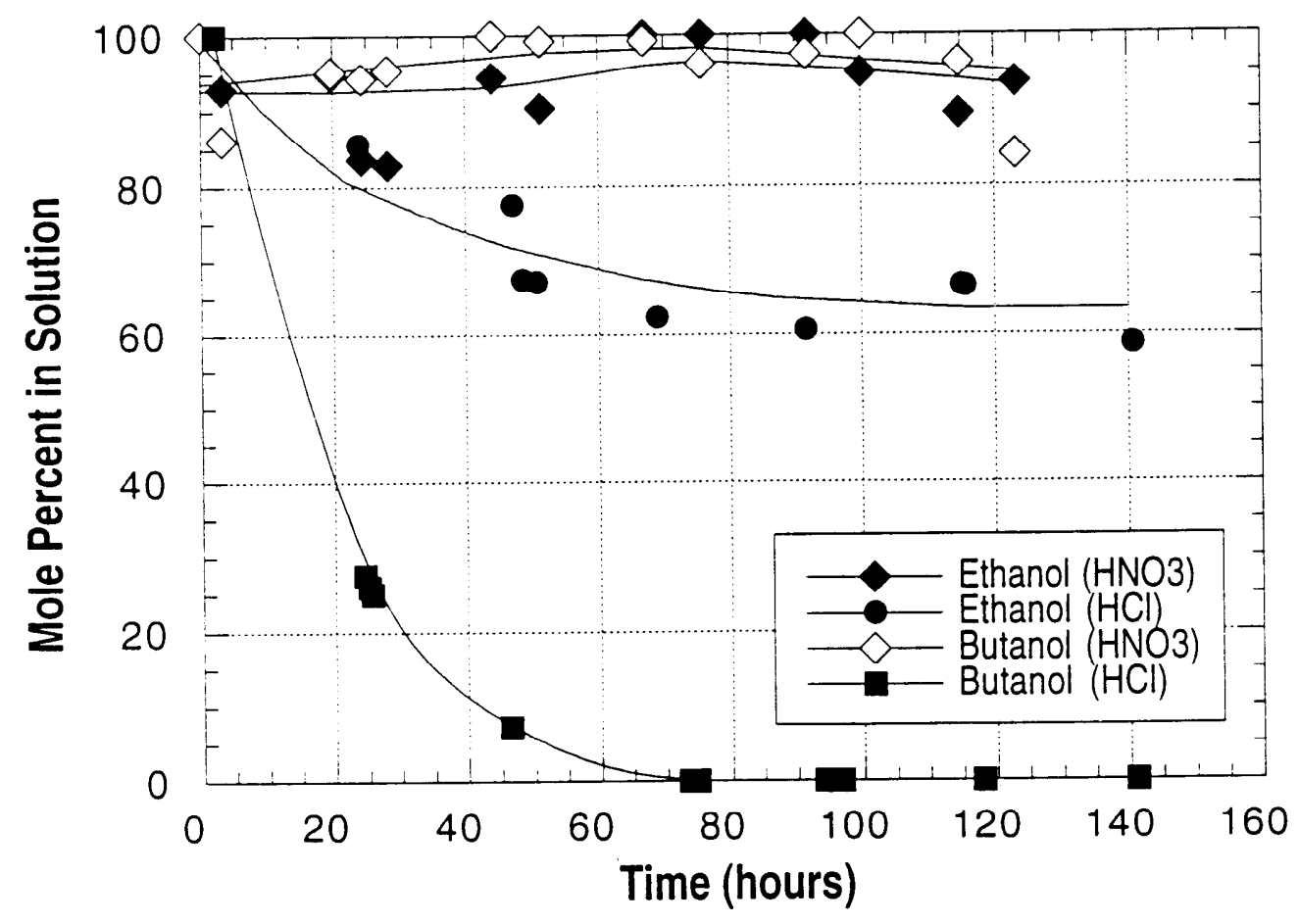

